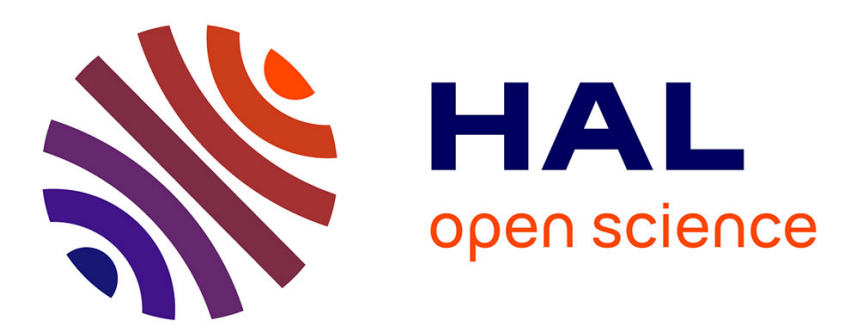

\title{
Selecting radiomic features from FDG-PET images for cancer treatment outcome prediction
}

\author{
Chunfeng Lian, Su Ruan, Thierry Denoeux, Fabrice Jardin, Pierre Vera
}

\section{To cite this version:}

Chunfeng Lian, Su Ruan, Thierry Denoeux, Fabrice Jardin, Pierre Vera. Selecting radiomic features from FDG-PET images for cancer treatment outcome prediction. Medical Image Analysis, 2016, 32, pp.257-268. 10.1016/j.media.2016.05.007 . hal-01324553

\section{HAL Id: hal-01324553 \\ https://hal.science/hal-01324553}

Submitted on 1 Jun 2016

HAL is a multi-disciplinary open access archive for the deposit and dissemination of scientific research documents, whether they are published or not. The documents may come from teaching and research institutions in France or abroad, or from public or private research centers.
L'archive ouverte pluridisciplinaire HAL, est destinée au dépôt et à la diffusion de documents scientifiques de niveau recherche, publiés ou non, émanant des établissements d'enseignement et de recherche français ou étrangers, des laboratoires publics ou privés. 


\title{
Selecting Radiomic Features from FDG-PET Images for Cancer Treatment Outcome Prediction
}

\author{
Chunfeng Lian ${ }^{\mathrm{a}, \mathrm{b}}$, Su Ruan ${ }^{\mathrm{b}}$, Thierry Denœux ${ }^{\mathrm{a}}$, Fabrice $\operatorname{Jardin}^{\mathrm{b}, \mathrm{d}}$, \\ Pierre Vera ${ }^{\mathrm{b}, \mathrm{c}}$ \\ ${ }^{a}$ Sorbonne Universités, Université de Technologie de Compiègne, CNRS, UMR 7253 \\ Heudiasyc, France \\ ${ }^{b}$ Université de Rouen, QuantIF, EA 4108 LITIS, France \\ ${ }^{c}$ Centre Henri-Becquerel, Department of Nuclear Medicine, France \\ ${ }^{d}$ Centre Henri-Becquerel, Department of Hematology, France
}

\begin{abstract}
As a vital task in cancer therapy, accurately predicting the treatment outcome is valuable for tailoring and adapting a treatment planning. To this end, multisources of information (radiomics, clinical characteristics, genomic expressions, etc) gathered before and during treatment are potentially profitable. In this paper, we propose such a prediction system primarily using radiomic features (e.g., texture features) extracted from FDG-PET images. The proposed system includes a feature selection method based on Dempster-Shafer theory, a powerful tool to deal with uncertain and imprecise information. It aims to improve the prediction accuracy, and reduce the imprecision and overlaps between different classes (treatment outcomes) in a selected feature subspace. Considering that training samples are often small-sized and imbalanced in our applications, a data balancing procedure and specified prior knowledge are taken into account to improve the reliability of the selected feature subsets. Finally, the Evidential K-NN (EK-NN) classifier is used with selected features to output prediction results. Our prediction system has been evaluated by synthetic and clinical datasets, consistently showing good performance.

Keywords: Dempster-Shafer theory, feature selection, imbalanced learning, outcome prediction, cancer, PET images
\end{abstract}

Preprint submitted to Medical Image Analysis

15 mai 2016 


\section{Introduction}

Accurate outcome prediction prior to or even during cancer therapy is of great clinical value. It benefits the adaptation of more effective treatment planning for individual patient. With the advances in medical imaging technology,

5 radiomics, referring to the extraction and analysis of a large amount of quantitative image features, provide an unprecedented opportunity to improve personalized treatment assessment (Aerts et al., 2014). Positron emission tomography (PET), with the radio-tracer fluoro-2-deoxy-D-glucose (FDG), is one of the important and advanced imaging tools for diagnosis, staging, and restaging of cancers. According to practice guidelines presented by the Society of Nuclear Medicine and Molecular Imaging (SNMMI) 1 . FDG-PET or FDG-PET/CT is now playing an essential role in clinical oncology, such as initial staging and gross tumor volume delineation for lung cancer patients receiving radiotherapy; initial staging and restaging of esophageal cancer; and routine pre-treatment staging and restaging of patients with Hodgkin lymphoma and many subtypes of non-Hodgkin lymphoma, etc.

Apart from diagnosis and staging, the functional information provided by FDG-PET has also emerged to be predictive of the pathologic response of a treatment in some types of cancers, such as lung tumor, esophageal tumor (Tan 20 et al. 2013) and cervix tumor (Barwick et al. 2013). For this application, variety radiomic features are well-explored on FDG-PET (Cook et al., 2014), which include standardized uptake values (SUVs), e.g., $\mathrm{SUV}_{\text {max }}, \mathrm{SUV}_{\text {peak }}$ and $\mathrm{SUV}_{\text {mean }}$, to describe metabolic uptakes in a region of interest (ROI), and metabolic tumor volume (MTV) and total lesion glycolysis (TLG) to describe metabolic tumor burdens. Apart from SUV-based features, some complementary characterization of PET images, like texture analysis (Tixier et al., 2011) and shape analysis (El Naqa et al., 2009), may also provide supplementary knowledge associated with the treatment outcome. Although the quantification of these

1. http ://www.snmmi.org/ClinicalPractice/ 
radiomic features, as well as the calculation of their temporal changes during the treatment, have been claimed to have the discriminative power (Aerts et al. 2014), the solid application is still hampered by some practical difficulties :

First, abounding features (e.g., radiomics and clinical characteristics) can be collected for outcome prediction, but without any consensus to determine the most discriminative factors among them. Thus, finding information regarding 35 the most predictive features could be interesting from the point of clinicians.

Second, comparing to a relatively large amount of input features, only a limited number of observations (small data size is often encountered in the medical domain) are available for constructing a prediction system. A high dimensional feature space may increase the complexity of the learning models, thus leading 40 to high risk of over-fitting on the small-sized learning set.

Third, it often happens that some of the input features are irrelevant with the outcome label. Moreover, badly defined features sometimes may even degrade the performance of a prediction model.

Feature selection is a feasible solution for above challenges. It aims to select a subset of features that can facilitate data interpretation and improve prediction accuracy (Guyon and Elisseeff, 2003). Univariate selection and multivariate selection are two rough categories of feature selection algorithms. According to chosen statistical measures, univariate methods utilize variable ranking as the principal selection mechanism. RELIEF (RELevance In Estimating

50 Features) (Kira and Rendell, 1992) is considered as one of the most successful univariate selection methods, in which a margin-based criterion is used to rank the features. FAST (Feature Assessment by Sliding Thresholds) Chen and Wasikowski, 2008), another feature ranking method, has the ability to tackle small sample size and imbalanced data problems. These univariate algorithms are simple and scalable; however, they may produce sub-optimal subsets as they ignore the interaction between features (Guyon and Elisseeff, 2003).

Different from ranking features, multivariate methods evaluate a subset of features ensemble. Sequential Forward Selection (SFS) and Sequential Forward Floating Selection (SFFS) (Pudil et al. 1994) are two classical subset selec- 
tion methods. According to the prediction accuracy of a specific classifier, and starting from an empty set, SFS repeatedly selects the best feature among the remaining features to yield a nested feature subset. Since former included features can not be deleted anymore, it has the possibility to be trapped in local minima. SFFS has been used with learning methods to automatically detect lung nodules in thoracic CT (Murphy et al., 2009). It in some sense reduces the nesting problem of SFS, but still has the risk to be sub-optimal with limited learning instances (Mi et al. 2015). To improve the performance of forward selection methods (such as SFS and SFFS) on small-sized datasets, a Hierarchical Forward Selection (HFS) method with an advanced searching strategy was proposed by (Mi et al. 2015). Different with SFS, HFS retains all candidate feature subsets that improve the classification accuracy in each iteration. As the result, it is more likely to obtain the most discriminative feature subset, while with the cost of increased searching time. Based on a generalization of the Support Vector Machine (SVM), Guyon et al. embedded a Recursive Feature Elimination pro75 cedure into the construction of the SVM classifier (namely SVMRFE) (Guyon et al. 2002). The variants of this method have been successfully applied for prostate cancer volume estimation (Ou et al. 2009) and deformable registration in medical imaging (Ou et al. 2011). Starting with all input features, and before reaching a predefined number of remaining features, SVMRFE progres-

so sively eliminates the least relevant features. It yields nested feature subsets, and has the risk of removing useful features that are complementary to others. Kernel Class Separability (KCS)-based feature selection method ranks feature subsets according to the class separability (Wang, 2008). As a robust method, KCS has found promising application for tumor delineation in multi-spectral MRI images (Zhang et al., 2011). But just like univariate methods, a threshold should be manually specified for KCS to output a feature subset.

Apart from the prediction accuracy, the stability of feature selection is also an important issue. As pointed by (Somol and Novovicova, 2010), the stability of a feature selection algorithm, referring to its robustness against changing conditions (e.g., perturbations of training data), can directly effect the reliability 
of a learning system. A key issue of the conventional feature selection methods discussed above is the difficulty to ensure robust selection performance with severely imperfect knowledge, such as seriously imbalanced training set, and high overlapping or noisy training set.

95 ㄴ To learn efficiently from noisy and high overlapping training dataset, Lian et al. 2015a proposed a robust subset selection method, namely Evidential Feature Selection (EFS), based on the Dempster-Shafer Theory (DST) (Shafer, 1976), a powerful tool for modeling and reasoning with uncertain and/or imprecise information. This method allows to quantify the uncertainty and imprecision resulted by different feature subsets. A specific loss function with a sparsity constraint is minimized to find a required subset that leads to both high classification accuracy and small overlaps between different classes. Due to system noise and low-resolution of PET imaging, as well as the effect of small tumor volumes (Brooks and Grigsby, 2014), in our application, the training set used for constructing the prediction system may contain imprecise or inaccurate observations. Under this condition, EFS can provide better performance than other conventional methods (Lian et al., 2015b). However, the imbalanced learning problem in feature selection (another important issue of medical data) is still left unsolved for this method.

${ }_{110}$ In this paper, we propose a new framework based on our previous work (EFS) for PET imaging based treatment outcome prediction. To this end, a data balancing procedure is added to EFS, so as to control the influence of imbalanced learning data on feature selection. In addition, to cope with small-sized datasets and to improve the subset robustness, prior knowledge is included in EFS to guide the feature selection procedure. The loss function used in the original EFS is also changed to reduce the complexity of the prediction system. Finally, the Evidential K-NN (EK-NN) rule (Denœux, 1995), a stable classification method based on DST, is used with selected feature subsets to output prediction results.

The rest of this paper is organized as follows. The background on DST 120 and the original EFS is recalled in Section 2, Then, an improved EFS with prior knowledge and data balancing is introduced in Section 3 . The proposed 
method is evaluated by three clinical datasets described in Section 4 , and the experimental results are summarized in Section 5. Some discussions and the conclusion are presented in Section 6 and Section 7 , respectively.

The necessary background on DST and the original EFS is briefly reviewed in Sections 2.1 and 2.2, respectively.

\subsection{Dempster-Shafer Theory}

DST is also known as the theory of belief functions or Evidence theory. As an extension of probability theory and the set-membership approach, DST has shown remarkable applications in divers fields, such as medical image processing (Bloch, 1996, Lelandais et al., 2014; Makni et al., 2014), statistical machine learning (Zhu and Basir, 2005; Denœux and Smets, 2006; Masson and Denœux, 2008: Liu et al., 2015), and computer vision (Xu et al., 2014: Wang et al., 2014)

35 etc. DST consists of two main components, i.e., the quantification of a piece of evidence and the combination of different items of evidence.

\subsubsection{Evidence Quantification}

DST is a formal framework for reasoning under uncertainty based on the modeling of evidence (Shafer, 1976). Let $\omega$ be a variable taking values in a finite domain $\Omega=\left\{\omega_{1}, \cdots, \omega_{c}\right\}$, called the frame of discernment. An item of evidence regarding the actual value of $\omega$ can be represented by a mass function $m$ on $\Omega$, defined from the powerset $2^{\Omega}$ to the interval $[0,1]$, such that

$$
\sum_{A \subseteq \Omega} m(A)=1
$$

Each number $m(A)$ denotes a degree of belief attached to the hypothesis that $\omega \in A$. Any subset $A$ with $m(A)>0$ is called a focal element of mass function $m$. Function $m$ is said to be normalized if $m(\emptyset)=0$. Corresponding to a normalized 
mass function $m$, we can associate belief and plausibility functions from $2^{\Omega}$ to $[0,1]$, which are defined as :

$$
\operatorname{Bel}(A)=\sum_{B \subseteq A} m(B) ; \quad P l(A)=\sum_{B \cap A \neq \emptyset} m(B) .
$$

Quantity $\operatorname{Bel}(A)$ can be interpreted as the degree to which the evidence supports the hypothesis $\omega \in A$, while $P l(A)$ can be interpreted as the degree to which the evidence is not contradictory to that hypothesis. Functions $\mathrm{Bel}$ and $\mathrm{Pl}$ are linked by the relation $\operatorname{Pl}(A)=1-\operatorname{Bel}(\bar{A})$. They are in one-to-one correspondence with mass function $m$.

\subsubsection{Evidence Combination}

In DST, beliefs are refined by aggregating different items of evidence. Dempster's rule of combination (Shafer, 1976), as well as its unnormalized version, i.e., the conjunctive combination rule defined in the Transferable Belief Model (TBM) (Smets and Kennes, 1994), are basic mechanisms for evidence fusion.

Let $m_{1}$ and $m_{2}$ be two mass functions derived from two independent items of evidence. They can be fused via the TBM conjunctive rule to induce a new mass function $m_{1} @ 2$ defined as

$$
m_{1} \bigcirc_{2}(A)=\sum_{B \cap C=A} m_{1}(B) m_{2}(C) .
$$

This new mass function reduces uncertainty and imprecision via transferring masses of belief to conjunctions of the focal elements. Quantity $m_{1} @ 2(\emptyset)$ measures the degree of conflict between evidence $m_{1}$ and $m_{2}$. If $m_{1} \bigcap_{2}(\emptyset)<1$, the new mass function obtained by Dempster's rule can be represented as

$$
m_{1 \oplus 2}(A)= \begin{cases}0 & \text { if } A=\emptyset, \\ \frac{m_{1} @_{2}(A)}{1-m_{1} \bigodot_{2}(\emptyset)} & \text { otherwise. }\end{cases}
$$

As can be seen, Dempster's rule normalizes the conflict obtained by the TBM conjunctive rule. Both the TBM conjunctive rule and Dempster's rule are commutative and associative. They can be easily generalized to combine $N(\geq 2)$ independent sources of information. 


\subsection{Evidential Feature Selection}

Let $\left\{\left(X_{i}, Y_{i}\right) \mid i=1, \cdots, N\right\}$ be a collection of $N$ training pairs, in which $X_{i}=\left[x_{i, 1}, \cdots, x_{i, V}\right]^{T}$ is the $i$ th training instance with $V$ features, and $Y_{i} \in$ $\left\{\omega_{1}, \cdots, \omega_{c}\right\}$ is the corresponding class label.

EFS (Lian et al., 2015a) searches for a qualified feature subset according to three requirements : first, high classification accuracy; second, low imprecision and uncertainty, namely small overlaps between different classes in the output feature space; third, sparsity to reduce the risk of over-fitting. To learn such a feature subset, EFS uses a weighted Euclidian distance measure to represent the dissimilarity between any two training instances. Hence, the dissimilarity between $X_{i}$ and $X_{j}$ is

$$
d_{i, j}=\sqrt{\sum_{p=1}^{V} \lambda_{p} d_{i j, p}{ }^{2}},
$$

where $d_{i j, p}=\left|x_{i, p}-x_{j, p}\right|$ represents the difference between the $p$ th dimension of $X_{i}$ and $X_{j}$. Features are selected via changing the value of the binary vector $\Lambda=\left[\lambda_{1}, \ldots, \lambda_{V}\right]^{T}$. As the result, the $p$ th dimension of the input feature space is selected when $\lambda_{p}=1$, while eliminated when $\lambda_{p}=0$.

We orderly regard each training instance $X_{i}$ as a query object. Then, other samples in the training pool can be considered as independent items of evidence that support different hypotheses regarding the class membership of $X_{i}$. The evidence offered by the training sample $\left(X_{j}, Y_{j}=\omega_{q}\right)$ is partially reliable, and can be modeled by a mass function

$$
\left\{\begin{array}{l}
m_{i, j}\left(\left\{\omega_{q}\right\}\right)=e^{-\gamma_{q} d_{i, j}^{2}}, \\
m_{i, j}(\Omega)=1-e^{-\gamma_{q} d_{i, j}^{2}},
\end{array}\right.
$$

where $d_{i, j}$ is the distance between $X_{i}$ and $X_{j}$ that measured by (5). Positive parameters $\gamma=\left[\gamma_{1}, \ldots, \gamma_{c}\right]^{T}$ are set as the inverse of the mean distance between training instances from the same class.

After obtaining all the independent mass functions for $X_{i}$, they can be further fused by a mixed combination rule, called Dempster+Yager rule (Lian et al. 165 2015a), so as to obtain a global one describing the class membership of $X_{i}$. This 
rule consists of two main steps : first, using Dempster's rule to combine mass functions originated from the same class, and discounting the resulting mass function according to the number of instances in this class; second, combining the discounted mass functions originated from different classes via the Yager's rule (Yager, 1987) to output the global mass function. This combination procedure integrates the advantages of Dempster's and Yager's rules, thus could robustly represent all imprecision and uncertainty of the training data on the whole frame of discernment (i.e., $\Omega$ ).

Finally, based on the global mass functions for all training samples, a loss function with respect to the binary vector $\Lambda=\left[\lambda_{1}, \ldots, \lambda_{V}\right]^{T}$ is constructed for feature selection,

$$
\arg \min _{\Lambda} \frac{1}{N} \sum_{i=1}^{N} \sum_{q=1}^{c}\left\{P l_{i}\left(\left\{\omega_{q}\right\}\right)-t_{i, q}\right\}^{2}+\frac{1}{N} \sum_{i=1}^{N} m_{i}(\Omega)+\beta\|\Lambda\|_{0},
$$

where $m_{i}$ and $P l_{i}$, concerning $\Lambda$, are the global mass function and the corresponding plausibility function of the training instance $X_{i}$. The first term of (7) is a mean squared error measure corresponding to the first requirement of EFS (namely high classification accuracy). Binary vector $t_{i}$ is the class label indicator, with $t_{i, q}=\delta_{i, q}$ if and only if $Y_{i}=\omega_{q}$. The second term of (7) penalizes feature subsets that lead to high overlaps between different classes, thus corresponding to the second requirement of EFS. The last term, which is an approximation of the $l_{0}$-norm of $\Lambda$, forces the selected features to be sparse, thus realizing the last requirement of EFS. Parameter $\beta$ controls the influence of this sparsity penalty.

The mixed combination rule used in the original EFS can lead to robust quantification of data uncertainty and imprecision. However, since a discounting procedure is included, additional parameters increase method's complexity. To cope with this problem, we propose a new method described in the next section. 


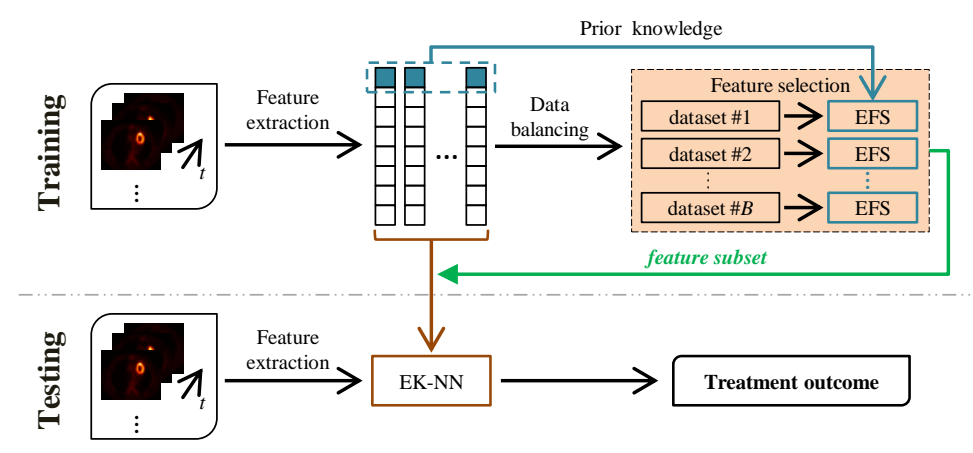

Figure 1: Protocol of the prediction system.

\section{Method}

The proposed prediction system is learnt on a dataset $\left\{\left(X_{i}, Y_{i}\right) \mid i=1, \cdots, N\right\}$ Finally, based on the selected feature subset, the Evidential $K$-Nearest-Neighbor 
(EK-NN) classification rule is trained with the original training dataset to predict the cancer treatment outcome.

\subsection{Feature Extraction}

To extract features, FDG-PET images for the same patient acquired at different time points are registered to the baseline image (i.e., image at initial staging) with a rigid registration method. The registration result is manually adjusted by physicians to avoid obvious misregistration. The ROIs around tumors are delineated by a relative threshold method, or manually delineated by experienced physicians when the result obtained by the threshold method is not reliable. It is worth to mention that the reproducibility of the manual tumor delineation has been evaluated in some clinical studies (Lemarignier et al., 2014). Three types of PET imaging features are quantified, namely SUV-based features, texture features, and the temporal changes of these two types of features.

$S U V$-based features. Five types of SUV-based features are calculated from the ROI of each PET stack, namely $\mathrm{SUV}_{\text {min }}, \mathrm{SUV}_{\text {max }}, \mathrm{SUV}_{\text {peak }}, \mathrm{MTV}$ and TLG. The detail description of these features, and the formulas for calculating them are shown in the Appendix (Table A.7).

225 Texture features. To characterize tumor uptake heterogeneity, texture features are also considered in our prediction system. As has been claimed to be effective in PET image characterization (Tixier et al., 2011), Gray Level Size Zone Matrix (GLSZM) (Thibault et al., 2014) is used to extract texture features. To this end, we resample voxel intensities inside the ROI to $2^{3}$ different values. By defining the connected voxels with the same gray level as a zone, a matrix with $2^{3}$ rows is then deduced, in which the element at row $r$ and column $s$ stores the number of zone with gray level $r$ and size $s$. The number of columns of this matrix is determined by the size of the largest zone. Therefore, a wide and flat matrix indicates that the texture information is homogeneous in the predefined ROI; while heterogeneity when the matrix is narrow. Based on this matrix, 
we compute eleven variables to describe the regional heterogeneity. The formulas for calculating these GLSZM-based features are presented in the Appendix (Table A.8).

Temporal changes of image features. Considering that the temporal changes of value, we propose to calculate their relative difference between the baseline and the follow-up PET acquisitions as additional features. The relative difference can be generally represented as $\Delta f=\left(f_{t}-f_{0}\right) / f_{0}$, where $f_{0}$ and $f_{t}$ denote the same kind of feature extracted from the baseline and the follow-up images,

3.3. Improved EFS

To reduce the complexity of the original EFS, a new criterion is constructed for feature selection.

Assuming $X_{i}$ is a query pattern, other samples in the training pool can be regarded as independent evidence regarding the outcome label of patient $i$. As 255 discussed in Section 2.2 the evidence offered by each training instance $X_{j}(\neq i)$ can be quantified as a mass function using (6) and (5). Since this mass function provides little information when $d_{i, j}$ is too large $\left(m_{i, j}(\Omega) \approx 1\right)$, it is sufficient to just consider the mass functions offered by the first $K$ (with a large value, e.g., $\geq 10$ ) nearest neighbors of each query pattern $X_{i}$.

Let $\left\{X_{i_{1}}, \ldots, X_{i_{K}}\right\}$ be the selected training samples for $X_{i}$. Correspondingly, $\left\{m_{i, i_{1}}, \ldots, m_{i, i_{K}}\right\}$ are their mass functions. We assign $\left\{X_{i_{1}}, \ldots, X_{i_{K}}\right\}$ into two different groups $\left(\Theta_{1}\right.$ and $\left.\Theta_{2}\right)$ according to their outcome labels. In each group with the same outcome label, the TBM conjunctive rule (3) is used to combine 
the corresponding mass functions. Hence, when $\Theta_{q} \neq \emptyset(q=1$ or 2$)$, the resulting mass function $m_{i}^{\Theta_{q}}$ can be represented as

$$
\left\{\begin{array}{l}
m_{i}^{\Theta_{q}}\left(\left\{\omega_{q}\right\}\right)=1-\prod_{X_{i_{p}} \in \Theta_{q}}^{p=1, \ldots}\left(1-e^{-\gamma_{q} d_{i, i_{p}}^{2}}\right), \\
m_{i}^{\Theta_{q}}(\Omega)=\prod_{X_{i_{p}} \in \Theta_{q}, \ldots, K}^{p, K}\left(1-e^{-\gamma_{q} d_{i, i_{p}}^{2}}\right)
\end{array}\right.
$$

while, when $\Theta_{q}$ is empty, $m_{i}^{\Theta_{q}}(\Omega)=1$. After that, mass functions $m_{i}^{\Theta_{1}}$ and $m_{i}^{\Theta_{2}}$ are further combined via the TBM conjunctive rule, so as to obtain a global mass function $M_{i}$ regarding the class membership of $X_{i}$,

$$
\begin{cases}M_{i}\left(\left\{\omega_{1}\right\}\right) & =m_{i}^{\Theta_{1}}\left(\left\{\omega_{1}\right\}\right) \cdot m_{i}^{\Theta_{2}}(\Omega) \\ M_{i}\left(\left\{\omega_{2}\right\}\right) & =m_{i}^{\Theta_{2}}\left(\left\{\omega_{2}\right\}\right) \cdot m_{i}^{\Theta_{1}}(\Omega) \\ M_{i}(\Omega) & =m_{i}^{\Theta_{1}}(\Omega) \cdot m_{i}^{\Theta_{2}}(\Omega) \\ M_{i}(\emptyset) & =m_{i}^{\Theta_{1}}\left(\left\{\omega_{1}\right\}\right) \cdot m_{i}^{\Theta_{2}}\left(\left\{\omega_{2}\right\}\right) .\end{cases}
$$

the influence of the sparsity penalty. It should be tuned specifically be a rough gird search strategy. 
Considering that the solution of 10 is integer constrained (vector $\Lambda$ should be binary), an integer Genetic Algorithm (GA), namely the MI-LXPM (Deep

More specifically, a feature ranking method, namely RELIEF (Kira and Rendell, 1992), is used to rank all kinds of SUV-based features. Let $\tilde{f}$ be a SUV-based feature that exists in each instance $X_{i}, \forall i \in\{1, \ldots, N\}$. RELIEF assigns a score $S(\tilde{f})$ to $\tilde{f}$ in the form of

$$
S(\tilde{f})=\frac{1}{N} \sum_{i=1}^{N}\left(\frac{1}{k} \sum_{j=1}^{k} \operatorname{diff}\left(\tilde{f}, X_{i}, \operatorname{miss}_{j}^{i}\right)-\frac{1}{k} \sum_{j=1}^{k} \operatorname{diff}\left(\tilde{f}, X_{i}, h i t_{j}^{i}\right)\right),
$$

where $h i t_{j}^{i}$ and $m i s s_{j}^{i}, j \in\{1, \ldots, k\}$, are the nearest neighbors of $X_{i}$ that originated from the same class and the opposite class, respectively. Function $\operatorname{dif} f\left(\tilde{f}, X_{1}, X_{2}\right)$ calculates the difference between the values of the feature $\tilde{f}$ for any two instances $X_{1}$ and $X_{2}$. The number of nearest neighbors (i.e. $k$ ) used in

(11) was always set to 5 in all our applications. 
The obtained score $S(\tilde{f})$ is directly proportional to the informativeness of the feature $\tilde{f}$. Therefore, the SUV-based feature with the largest score is included in EFS as a fixed element of the optimal feature subset. In other words, if the pre-determined feature $\tilde{f}$ is located in the first dimension of the input feature 300 space, the value of $\lambda_{1}$ is forced to be 1 (can not be 0) when minimizing (10). This added constraint drives EFS into a confined searching space. It ensures more robust feature selection, thus increasing the reliability of the prediction system.

\subsection{Data Balancing}

Ensemble with small training sample size, class imbalance is also a typical problem of medical data. Since most of the conventional feature selection methods are designed for well-balanced training data, the class imbalance problem could hinder them to obtain a qualified feature subset. For example, as selecting features according to the accuracy of a specific classifier, SFS and SFFS (Pudil et al. 1994) may output a feature subset that achieves high classification accuracy by simply assigning all training instances to the majority class.

Pre-sampling, either over-sampling the minority class or under-sampling the majority class, is a commonly used approach for the imbalanced learning problems. As a powerful method, Synthetic Minority Over-sampling TEchnique (SMOTE) can generalize the decision region of the minority class via generating synthetic examples (Chawla et al. 2002). It has shown plenty of successes in many applications, and its variants, such as ADAptive SYNthetic sampling (ADASYN) (He et al. 2009), can further improve the performance.

On this account, ADASYN is adopted in our prediction system to balance the training data for feature selection. The key idea of ADASYN is to adaptively create synthetic samples according to the distribution of the minority class instances, where more instances are generated for the minority class samples that have higher difficulty in learning. The level of difficulty in learning for each minority instance is measured with respect to the ratio of the majority class instances in its $k$-nearest-neighborhood ( $k$ was set to 5 in all our applications). 


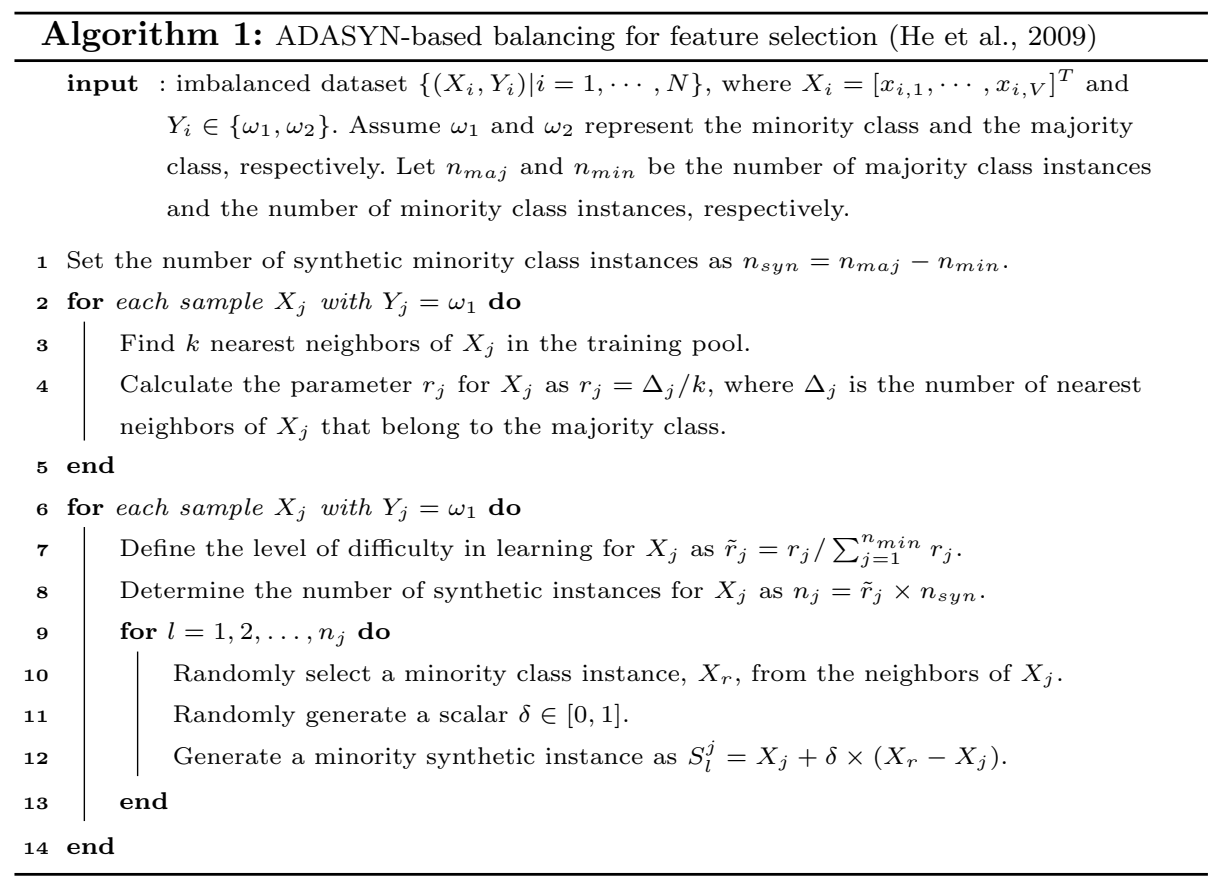

Given an imbalanced training dataset, ADASYN outputs an balanced training dataset via the procedure summarized in Algorithm 1 . However, due to the random nature of the data balancing procedure, and also with a limited number of training samples, the balanced training dataset obtained by Algorithm 1 can not always be more representative than the original training dataset. Therefore, in our prediction system, ADASYN is totally executed $B(>1)$ times to provide $B$ balanced training datasets. EFS is then executed with these balanced datasets to obtain $B$ feature subsets. The final output is determined as the most frequently subset that occurred in the $B$ independent actions.

\subsection{Classification}

Feature subsets selected by the improved EFS should be used with a classifier to predict the treatment outcome. To this end, case-based methods, such as the $K$-NN rules and the SVM classifier, are practically good alternatives thanks to their efficiency. As a stable method that offers global treatment of the imperfect knowledge regarding the training data, the EK-NN (Denœux, 1995) 
classification rule, developed in the DST framework, is selected as the default classifier in our prediction system. Parameters used in the EK-NN rule are optimized using the method proposed by (Zouhal and Denœux, 1998). It is worth to note that only the original training dataset with selected features are used to 345 train the classification rule (i.e., no synthetic instance is used during classification), since we assume that instances from the two different classes are widely separated in the feature subspace selected by the improved EFS, while the data balancing procedure has little influence on the classification performance under this circumstance.

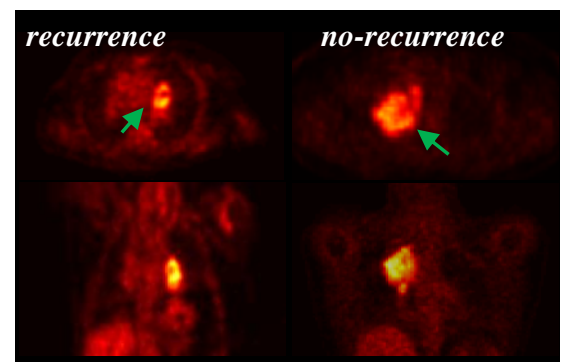

(a) Lung Tumors

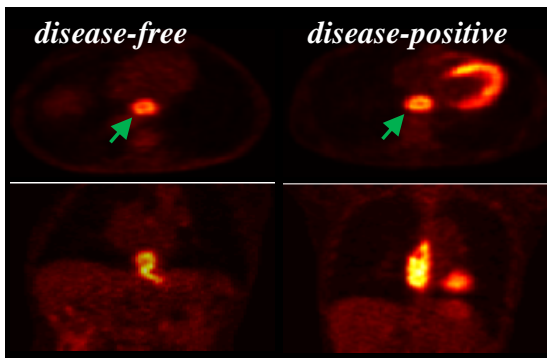

(b) Esophageal Tumors

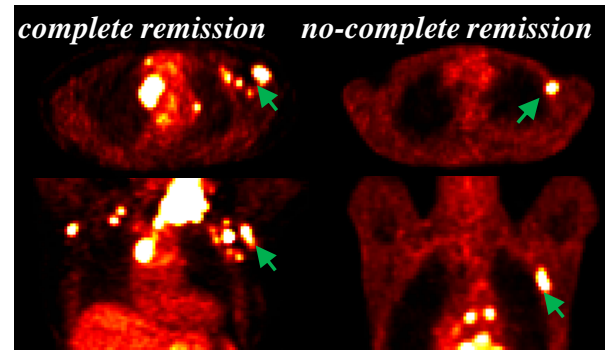

(c) Lymph Tumors

Figure 2: FDG-PET uptakes at tumor staging. For each dataset, two examples with different outcome labels are presented from two complementary views ( $x y$-plane and $x z$-plane); The arrows point out the tumor locations.

\section{Clinical Datasets}

The prediction system proposed in this paper has been evaluated by three real-world datasets. 
1) Lung Cancer Data. A cohort of twenty-five patients with inoperable stage II or III non-small cell lung cancer (NSCLC), treated with curative-intent chemoradiotherapy (CRT) or radiotherapy (RT). This dataset was extracted from three prospective studies (Calais et al., 2015). The total dose of included RT was 60-70 Gy, delivered in daily fractions of 2 Gy and five days a week. Each patient had histological proof of invasive NSCLC, and also had evaluable tumor lesions according to the Response Evaluation Criteria in Solid Tumors (RECIST 1.1). Initial tumor staging was performed based on fibreoptic bronchoscopy, CT scan, pulmonary function tests and biopsy. All patients also underwent FDGPET scans at initial staging (i.e., $\mathrm{PET}_{0}$, the baseline). The following PET scans for the same patient were acquired using the same device and under the same operational conditions. The first FDG-PET/CT acquisition $\left(\mathrm{PET}_{1}\right)$ was obtained after induction chemotherapy and before RT, followed by the second FDG-PET/CT scan $\left(\mathrm{PET}_{2}\right)$ performed during the fifth week of RT (approximately at a total dose of 40-45 Gy). The treatment response was systematically evaluated and followed-up at three months and one year after RT, or if there was a suspicious relapse. The endpoint was local/distant relapse (LR/DR) vs. complete response (CR) at one year, which was primarily defined by clinical evaluation and CT according to RECIST 1.1, and supplemented by FDG-PET/CT and fiberscope. Finally, nineteen LR/DR patients were grouped into the recurrence class (majority class), while the remaining six CR patients were labeled as no-recurrence (minority class).

2) Esophageal Cancer Data. A cohort of thirty-six patients with histologically confirmed esophageal squamous cell carcinomas, treated with definitive CRT according to the Herskovic scheme. This dataset was extracted from a retrospective clinical trial (Lemarignier et al., 2014). The included RT delivered 2 Gy per fraction per day, five sessions per week for a total of 50 Gy over five weeks. The initial tumor staging was performed based on oesophagoscopy with biopsies, CT scan, and endoscopic ultrasonography. Each patient also underwent a FDG-PET/CT scan at initial tumor staging, but the following PET scans 
were not complete for all the thirty-six patients. The patients were systematically evaluated and followed-up in a long term up to five years. According to RECIST 1.1 criteria, the response assessment performed one month after CRT was based on clinical evaluation and CT, and possibly supplemented by FDG$\mathrm{PET} / \mathrm{CT}$, and oesophagoscopy with biopsies. Thirteen patients were grouped to the disease-free class (minority class), since neither locoregional nor distant disease was detected on them; the remaining twenty-three patients were labeled as disease-positive (majority class).

3) Lymph Cancer Data. A cohort of forty-five patients with diffuse large B-cell lymphoma (DLBCL), treated with rituximab and a cyclophosphamide, doxorubicin, vincristine and prednisone $(\mathrm{CHOP}) / \mathrm{CHOP}$-like regimen. This dataset was the same as that in (Lanic et al. 2012). Each patient underwent FDG-PET scans before the onset of chemotherapy $\left(\mathrm{PET}_{0}\right)$ and also after three/four cycles of chemotherapy $\left(\mathrm{PET}_{1}\right)$. At least three weeks after the end of chemotherapy, the treatment response was evaluated according to the International Workshop Criteria (IWC) for non-Hodgkin lymphoma (NHL) response and according to IWC+PET. Thirty-nine patients were observed complete remission (majority class); while, the remaining six patients with refractory or partial response were grouped to the class non-complete remission (minority class).

For each dataset, PET image examples acquired at tumor staging are presented in Figure 2 .

Feature Description. As discussed in Section 3.2, three types of PET image features (SUV-based features, texture features and the temporal changes of them) were extracted. Apart from these image features, variables extracted from other sources of information are also potentially predictive factors. For the esophageal tumor dataset, since only PET images before the treatment were available, some clinical characteristics (patient gender, tumor stage, tumor location, dysphagia grade, etc) were included as the complementary knowledge. In the lymph tumor dataset, only four PET image features were available. As the supplementary information for them, eighteen genes related to the tumor subtype classification, 
and five genes related to the glucose transportation were also gathered according to the molecular analysis (Lanic et al. 2012). The three clinical datasets are briefly summarized in Table 1 where the number of features and the number of instances are presented. In addition, let the minority (majority) class be the positive (negative) class, we defined the imbalance ratio as $r=N_{p} /\left(N_{p}+N_{n}\right)$, where $N_{p}$ and $N_{n}$ are the number of positive and negative samples, respectively.

TABLE 1: Description of the three clinical datasets.

\begin{tabular}{cccc}
\hline dataset & sample size & feature size & imbalance ratio \\
\hline lung tumor & 25 & 52 & 0.24 \\
esoph. tumor & 36 & 29 & 0.36 \\
lymph tumor & 45 & 27 & 0.13 \\
\hline
\end{tabular}

\section{Experimental Results}

The presented experiments consist of two parts. In the first part, the feature selection performance of the improved EFS was compared with the original EFS, and also compared with some other feature selection methods. In the second part, we assessed the predictive power of the selected feature subsets, and compared them with the predictors that have been proven to be discriminative in clinical studies (e.g., MTV or TLG at staging for the esophageal cancer dataset (Lemarignier et al., 2014)).

\subsection{Feature Selection Performance}

The improved EFS used in our prediction system was compared with seven other methods, namely two univariate methods (RELIEF and FAST) and five multivariate methods (SFS, SFFS, SVMRFE, KCS, and HFS). As discussed in Section 11, the univariate methods rank features according to their individual discriminative power, while the multivariate methods evaluate a subset of features ensemble according to the class separability for a predefined classifier. Because of a limited number of instances, and in order to perform a comprehen435 sive assessment, all the compared methods were evaluated by the Leave-One- 
Out-Cross-Validation (LOOCV), and also by the $.632+$ Bootstrapping, which ensures low bias and variance estimation (Efron and Tibshirani, 1997).

As one of the metrics used to evaluate the selection performance, the robustness of the selected feature subsets was measured by the relative weighted consistency (Somol and Novovicova, 2010). Its calculation is based on feature occurrence statistics obtained from all iterations of the LOOCV or the $.632+$ Bootstrapping. The value of the relative weighted consistency ranges between $[0,1]$, where 1 means all selected feature subsets are approximately identical, while 0 represents no intersection between them. Together with the subset robustness, the classification results obtained during feature selection were also used to assess the feature selection performance. As the most classical figure of merit used in general pattern classification applications, the average Accuracy was adopted, which is defined as

$$
\text { Accuracy }=\frac{T P+T N}{T P+T N+F P+F N},
$$

where TP (true positives), TN (true negatives), FP (false positives) and FN (false negatives) represent, respectively, correctly classified positive cases, correctly classified negative cases, incorrectly classified negative cases, and incorrectly classified positive cases. However, the simple Accuracy measure is not adequate in the context of clinical management, where the TP rate and the $\mathrm{TN}$ rate are more clinically relevant, particularly when instances from different classes are severely imbalanced. For instance, in cancer diagnosis, there are usually more benign examples (negative cases) than malignant examples (positive cases), while a FN decision (i.e., misclassifying malignant as benign) usually comes at greater costs than a FP decision (i.e., misclassifying benign as malignant). Therefore, to comprehensively assess the classification performance of the imbalanced learning problems, the Receiver Operating Characteristics (ROC) analysis, which was also utilized apart from the Accuracy measure, is more suitable. The ROC makes use of the TP rate and the FP rate, which are defined as

$$
T P_{\text {rate }}=\frac{T P}{T P+F N} ; \quad F P_{\text {rate }}=\frac{F P}{T N+F P} .
$$


Based on the ROC curve, the Area Under the Curve (AUC) was calculated as the complementary measure of the Accuracy in our applications (since all the three examples are imbalanced).

Parameters of all the methods used in sequel are summarized as below :

- For the improved EFS, the parameter $B$ was set to 5 . The hyper-parameter $\beta$ was determined by a rough grid search strategy according to the training performance. On average, good results were obtained with $\beta$ between $[0.01,0.07]$ for the lung and lymph tumor datasets, while between $[0.1,0.3]$ for the esophageal tumor dataset.

- The cutoff thresholds used in RELIEF, FAST and KCS to output selected features were changed from 0.5 to 0.9 . Then, the best feature subset was determined according to the average Accuracy. Similarly, the predefined number of selected features that used in SFS, SFFS and SVMRFE was changed from 1 to 5 to output a sparsity feature subset.

- In SFS, SFFS and HFS, the SVM classifier (gaussian kernel, $\sigma=1$ ) was chosen as the predefined classifier.

- All parameters used in HFS were the same as that in (Mi et al. 2015).

- For the compared feature selection methods, the SVM classifier (gaussian kernel, $\sigma=1$ ) was adopted to predict the outcome, as it is commonly used with the multivariate methods, and also often used in clinical studies. In our prediction system, the EK-NN classification rule (instead of the SVM classifier) was used with the EFS to predict the treatment outcome.

Evaluation by the LOOCV. The robustness of the selected feature subsets, the average Accuracy, the average AUC, and the average subset size for different methods are summarized in Table 2, where the results for all the input features (the SVM classifier was used) are also presented as the baselines for comparison. From Table 2 we can observe that the improved EFS (denoted as $i$ EFS) used 465 in our prediction system always led to robust feature subsets for all the three examples as compared to other methods. Furthermore, it had better (for the esophageal and lymph tumor datasets) or at least the same (for the lung tumor 
TABLE 2: Feature selection performance evaluated by the LOOCV. EFS represents our previous work (Lian et al. 2015a), while $i$ EFS denotes the improved EFS that proposed in this paper. "All" represents the results for all the input features (without selection).

\begin{tabular}{|c|c|c|c|c|c|c|c|c|c|c|}
\hline & \multicolumn{10}{|c|}{ Lung Tumor Data } \\
\hline & All & RELIEF & FAST & SFS & SFFS & SVMRFE & $\mathrm{KCS}$ & HFS & EFS & $i \mathrm{EFS}$ \\
\hline Robustness & - & 0.64 & 0.65 & 0.85 & 0.32 & 0.56 & 0.50 & $\underline{1.00}$ & 0.94 & $\underline{1.00}$ \\
\hline Accuracy & 0.76 & 0.72 & 0.76 & 0.88 & 0.80 & 0.76 & 0.84 & $\underline{1.00}$ & $\underline{1.00}$ & $\underline{1.00}$ \\
\hline $\mathrm{AUC}$ & 0.50 & 0.60 & 0.35 & 0.95 & 0.61 & 0.74 & 0.81 & $\underline{1.00}$ & $\underline{1.00}$ & $\underline{1.00}$ \\
\hline \multirow[t]{3}{*}{ Subset size } & 52 & 10 & 14 & 2 & 5 & 5 & 3 & 3 & 4 & 4 \\
\hline & \multicolumn{10}{|c|}{ Esophageal Tumor Data } \\
\hline & All & RELIEF & FAST & SFS & SFFS & SVMRFE & $\mathrm{KCS}$ & $\mathrm{HFS}$ & EFS & $i \mathrm{EFS}$ \\
\hline Robustness & - & 0.94 & 1.00 & 0.26 & 0.23 & 0.80 & 0.94 & 0.53 & 0.92 & $\underline{1.00}$ \\
\hline Accuracy & 0.64 & 0.56 & 0.64 & 0.64 & 0.58 & 0.72 & 0.69 & 0.72 & 0.83 & $\underline{0.89}$ \\
\hline $\mathrm{AUC}$ & 0.12 & 0.54 & 0.12 & 0.50 & 0.55 & $\underline{0.76}$ & 0.57 & 0.67 & 0.69 & $\underline{0.77}$ \\
\hline \multirow[t]{3}{*}{ Subset size } & 29 & 2 & 27 & 5 & 5 & 5 & 2 & 5 & 3 & 3 \\
\hline & \multicolumn{10}{|c|}{ Lymph Tumor Data } \\
\hline & All & RELIEF & FAST & SFS & SFFS & SVMRFE & $\mathrm{KCS}$ & HFS & EFS & $i \mathrm{EFS}$ \\
\hline Robustness & - & $\underline{1.00}$ & 0.85 & 0.72 & 0.34 & 0.64 & $\underline{1.00}$ & 0.90 & 0.57 & $\underline{0.95}$ \\
\hline Accuracy & 0.87 & $\underline{0.96}$ & 0.82 & 0.89 & 0.87 & 0.89 & $\underline{0.96}$ & 0.87 & 0.89 & $\underline{0.93}$ \\
\hline $\mathrm{AUC}$ & 0.50 & 0.68 & 0.26 & 0.65 & 0.29 & 0.83 & 0.68 & 0.36 & $\underline{0.92}$ & $\underline{0.95}$ \\
\hline Subset size & 27 & 1 & 5 & 2 & 5 & 5 & 1 & 4 & 4 & 4 \\
\hline
\end{tabular}

dataset) AUC as compared to other methods. While the Accuracy of the RELIEF and the KCS was slightly better than the proposed $i$ EFS for the lymph tumor dataset (difference of 0.03), the AUC obtained by our method was much better than other methods (minimum difference of 0.12 ) for this severely imbalanced example (imbalanced ratio $r=0.13$ ). Comparing the results obtained by the original EFS (Lian et al., 2015a) with the proposed $i$ EFS, it can be found that the data balancing procedure and the incorporated prior knowledge did improve the reliability (relating to robust feature selection) and accuracy (relating to the average Accuracy and AUC) of our prediction system.

Evaluation by the $.632+$ Bootstrapping. The number of Bootstrap samples was set to 100. The robustness of the selected feature subsets, the average Accuracy, the average AUC, and the average subset size are summarized in Table 3. ${ }_{480}$ Consistent with the results presented in Table 2 the robustness of the proposed $i$ EFS that evaluated by the bootstrapping was still better than other methods 
TABLE 3: Feature selection performance evaluated by the $.632+$ Bootstrapping. EFS represents our previous work (Lian et al. 2015a), while $i$ EFS denotes the improved EFS that proposed in this paper. "All" represents the results for all the input features (without selection).

\begin{tabular}{|c|c|c|c|c|c|c|c|c|c|c|}
\hline & \multicolumn{10}{|c|}{ Lung Tumor Data } \\
\hline & All & RELIEF & FAST & SFS & SFFS & SVMRFE & $\mathrm{KCS}$ & HFS & EFS & $i \mathrm{EFS}$ \\
\hline Robustness & - & 0.16 & 0.11 & 0.22 & 0.14 & 0.12 & 0.10 & 0.48 & 0.21 & 0.82 \\
\hline Accuracy & 0.85 & 0.82 & 0.82 & 0.80 & 0.80 & 0.84 & 0.83 & 0.85 & 0.81 & $\underline{0.94}$ \\
\hline $\mathrm{AUC}$ & 0.37 & 0.64 & 0.60 & 0.67 & 0.66 & 0.53 & 0.65 & 0.81 & 0.77 & 0.94 \\
\hline \multirow[t]{3}{*}{ Subset size } & 52 & 7 & 10 & 5 & 5 & 5 & 29 & 3 & 4 & 4 \\
\hline & \multicolumn{10}{|c|}{ Esophageal Tumor Data } \\
\hline & All & RELIEF & FAST & SFS & SFFS & SVMRFE & $\mathrm{KCS}$ & HFS & EFS & $i \mathrm{EFS}$ \\
\hline Robustness & - & 0.33 & 0.61 & 0.30 & 0.16 & 0.31 & 0.29 & 0.32 & 0.44 & 0.74 \\
\hline Accuracy & 0.74 & 0.69 & 0.74 & 0.69 & 0.66 & 0.74 & 0.69 & 0.74 & 0.77 & $\underline{0.83}$ \\
\hline AUC & 0.63 & 0.66 & 0.63 & 0.64 & 0.63 & 0.75 & 0.66 & 0.71 & 0.75 & $\underline{0.82}$ \\
\hline \multirow[t]{3}{*}{ Subset size } & 29 & 6 & 25 & 2 & 5 & 5 & 3 & 5 & 3 & 3 \\
\hline & \multicolumn{10}{|c|}{ Lymph Tumor Data } \\
\hline & All & RELIEF & FAST & SFS & SFFS & SVMRFE & $\mathrm{KCS}$ & HFS & EFS & $i \mathrm{EFS}$ \\
\hline Robustness & - & $\underline{0.56}$ & 0.19 & 0.25 & 0.15 & 0.37 & 0.33 & 0.43 & 0.32 & $\underline{0.64}$ \\
\hline Accuracy & 0.92 & 0.92 & 0.91 & 0.90 & 0.90 & 0.89 & $\underline{0.93}$ & 0.91 & 0.90 & $\underline{0.93}$ \\
\hline $\mathrm{AUC}$ & 0.62 & 0.75 & 0.63 & 0.73 & 0.67 & 0.78 & 0.77 & 0.78 & 0.82 & $\underline{0.92}$ \\
\hline Subset size & 27 & 4 & 15 & 1 & 5 & 5 & 2 & 3 & 4 & 4 \\
\hline
\end{tabular}

for all the three examples. In addition, it also led to the best AUC (especially for the lymph and lung tumor examples with severely imbalanced ratio) and the best Accuracy. Comparing the results shown in Table 3 with that in Table 2 , we can find that the performance of all the compared methods was declined when evaluated by the bootstrapping. This result is reasonable and foreseeable : Since all the three datasets are small-sized, and due to the random nature of the $.632+$ bootstrapping, many bootstrap samples may be greatly underrepresented for learning a qualified feature subset. However, it is also worth to note that the difference between the proposed $i$ EFS and other methods was increased under this circumstance, which in some sense confirmed the effectiveness of the proposed method.

Selected Feature Subsets. The most frequent feature subsets selected by the improved EFS were kept the same between the LOOCV and the .632+ Bootstrapping for all the three datasets. The detail of the selected features are summarized 
TABLE 4: The most stable feature subset for the lung tumor dataset.

\begin{tabular}{ll}
\hline Feature type & Feature description \\
\hline SUV-based feature & $\mathrm{SUV}_{\text {max }}$ extracted from $\mathrm{PET}_{2}$. \\
GLSZM-based feature & Change of gray-level-non-uniformity between $\mathrm{PET}_{2}$ and $\mathrm{PET}_{0}$. \\
GLSZM-based feature & Change of zone-percentage between $\mathrm{PET}_{1}$ and $\mathrm{PET}_{0}$. \\
GLSZM-based feature & Change of zone-percentage between $\mathrm{PET}_{2}$ and $\mathrm{PET}_{0}$. \\
\hline
\end{tabular}

TABLE 5: The most stable feature subset for the esophageal tumor dataset.

\begin{tabular}{ll}
\hline Feature type & Feature description \\
\hline SUV-based feature & TLG extracted from $\mathrm{PET}_{0}$. \\
Clinical characteristic & Tumor staging as II \\
Clinical characteristic & Patient gender \\
\hline
\end{tabular}

in Table 4 to Table 6, respectively. For the lung tumor (Table 4), the $\mathrm{SUV}_{\max }$ during the fith week of RT $\left(\mathrm{PET}_{2}\right)$ has also been proven to have significant predictive power in the clinical study (Calais et al. 2015) ; for the esophageal tumor (Table 5), the role of the TLG at tumor staging $\left(\mathrm{PET}_{0}\right)$ has been clinically validated in (Lemarignier et al. 2014); and for the lymph tumor (Table 6), the difference between the $\mathrm{SUV}_{\max }$ before chemotherapy $\left(\mathrm{PET}_{0}\right)$ and the $\mathrm{SUV}_{\max }$ after three/four cycles of chemotherapy $\left(\mathrm{PET}_{1}\right)$ has also been recognized as a variable being capable to predict outcome in (Lanic et al., 2012).

According to above analysis, we could say that the feature subsets determined by our method are in consistent with the predictors that have been verified in clinical studies. More importantly, other kinds of features selected in each subset can give complementary information for these existing measures to improve the prediction performance.

\subsection{Prediction Performance}

The improved EFS used in our prediction system has robust feature selection performance. To further evaluate the predictive power of these selected feature subsets, the EK-NN classifier with $K=\{1, \ldots, 15\}$ was orderly evaluated by the $.632+$ Bootstrapping. The number of Bootstrap samples was set to 100 . The prediction performance was compared with that obtained by all the input features, and also compared with that obtained by the existing measures (pre- 


\begin{tabular}{|c|c|}
\hline Feature type & Feature description \\
\hline SUV-based feature & Change of $\mathrm{SUV}_{\max }$ between $\mathrm{PET}_{1}$ and $\mathrm{PET}_{0}$. \\
\hline SUV-based feature & $\mathrm{SUV}_{\max }$ extracted from $\mathrm{PET}_{0}$ \\
\hline Gene expression & MME Gene that relates to tumor subtype. \\
\hline Gene expression & SLC2A5 Gene that relates to glucose transportation. \\
\hline
\end{tabular}

dictors) which have been clinically validated and discussed in the last part of Section 5.1. The average AUC with respect to different $K$ is shown in Figure 3 . where (a)-(c) correspond to the results for the lung tumor, esophageal tumor and lymph tumor dataset, respectively. As can be seen, the selected feature subsets (green line) always led to higher AUC than the input features (blue line) for all the three examples. In addition, they also outperformed the clinically validated predictors (orange line) that self-included in these selected feature subsets. It seems to imply that complementary predictors are well determined for these existing measures in our prediction system.

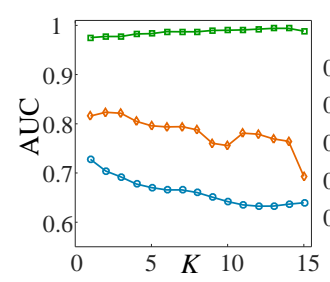

(a)

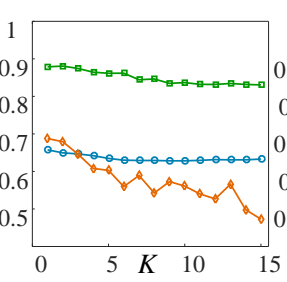

(b)

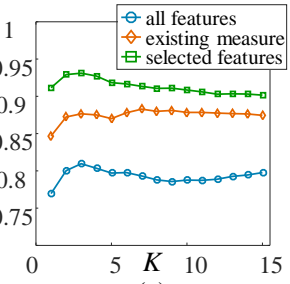

(c)

Figure 3: Prediction performance of the EK-NN classifier with respect to different $K$ : (a) lung tumor dataset, (b) esophageal tumor dataset, and (c) lymph tumor dataset. "all features", "selected features", and "existing measure" denote the results obtained by the input features, the selected feature subset and the predictor that has been clinically proven, respectively.

Misclassified instances. The main reason of misclassification is that the features extracted for these patients are located in the high-overlapping areas in the selected feature space, such as the boundary between two different classes. For the lung tumor dataset, only one patient, which belongs to the recurrence class, was often misclassified; For the lymph tumor dataset, only two instances 

tumor dataset was poorer than the other two examples, due to the lack of time dependent features extracted from the follow-up PET images.

\section{Discussion}

Influence of imbalance level. According to the analysis in Section 5.1, the competitiveness of the improved EFS seems to be strengthened when the dataset was highly imbalanced (e.g., the lymph tumor example). To support this finding, we further tested our method on a synthetic dataset with respect to different imbalance ratio $r \in\{0.1,0.2, \ldots, 0.5\}$. Both classes (positive or negative) of this synthetic dataset were generated by multivariate normal distributions. Assume that $\mu_{n}$ and $\mu_{p}$ are the mean vectors for the negative class and the positive class, respectively; while $\Sigma$ is the identical covariance matrix for both classes. To be consistent with our clinical examples, the values of $\mu_{n}, \mu_{p}$ and $\Sigma$ were directly copied as that of the lymph tumor dataset.

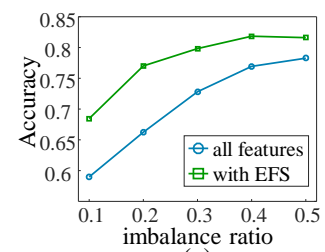

(a)

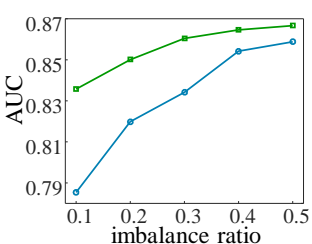

(b)

Figure 4: (a) Accuracy, and (b) AUC for the synthetic dataset.

Under each level of the imbalance ratio $r, 50$ samples were generated as a small-sized and imbalanced training dataset. After selecting features using the improved EFS, the EK-NN classifier was learnt to classify a balanced testing dataset. To minimize the uncertainty of the performance estimation, the balanced testing dataset consisted of 3000 test samples, and the evaluation was repeated 50 times for each level of $r$. The classification results with respect to different imbalance ratio are finally shown in Figure 4 . As can be seen, Accuracy and AUC obtained by the proposed method are better than directly using all 
the input features. In particular, the proposed method plays a significant role when the training dataset is severely imbalanced.

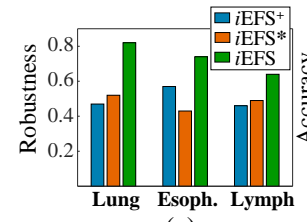

(a)

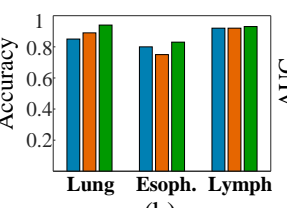

(b)

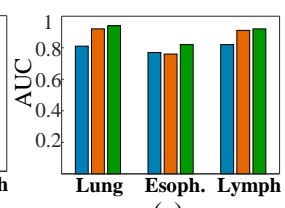

(c)

Figure 5: (a) Subset robustness, (b) Accuracy, and (c) AUC that evaluated by the .632+ Bootstrapping for the improved EFS without data balancing $\left(i \mathrm{EFS}^{+}\right)$, the improved EFS without prior knowledge $\left(i\right.$ EFS $\left.^{*}\right)$, and the improved EFS ( $i$ EFS), respectively.

Role of prior knowledge and data balancing. These two critical modules of our prediction system were successively removed to study the benefits of them. The performance that evaluated by the $.632+$ Bootstrapping (with 100 Bootstrap Samples) is shown in Figure 5 , in which $i$ EFS denotes the improved EFS used

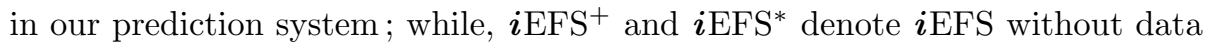
balancing and without prior knowledge, respectively. It can be found that both the included prior knowledge and the data balancing step are helpful for improving the selection performance and the prediction performance. When the dataset is severely imbalance (e.g., the lung tumor example), the data balancing procedure is especially significant for enhancing the robustness and the AUC.

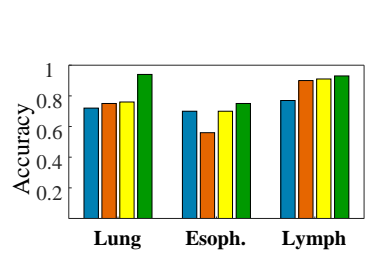

(a)

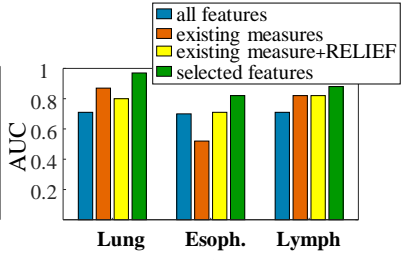

(b)

Figure 6: (a) Accuracy and (b) AUC of the logistic regression method that evaluated by the $.632+$ Bootstrapping. The selected features were compared with all the input features, the clinically validated predictors (i.e., existing measures), and the clinically validated predictors joint with features selected by the classical RELIEF (i.e., existing measure+RELIEF). 
Applicability of the improved EFS. To demonstrate whether the improved EFS gression, a well-established method widely used in clinical studies, was also adopted to classify the three tumor datasets with the feature subsets detailed in Table 4 to Table 6. The predictive power of the selected features was compared with that of all the input features, and that of the clinically validated predictors as the prior, the logistic regression joint with the classical RELIEF, involving to select features to combine with the clinically validated ones, was also presented as the basis for evaluation. Finally, results obtained by the $.632+$ Bootstrapping (with 100 Bootstrap samples) is summarized in Figure 6 based on which we may 575 say that the proposed method is not only useful for the DST-based classifiers, but also potentially helpful for other classifiers.

Multi-class problems. Apart from the binary-class examples discussed in this paper, the proposed method can also be easily generalized to handle the multiclass $(c \geq 2)$ problems. To this end, we need to replace $(9)$ with

$$
\begin{cases}M_{i}\left(\left\{\omega_{q}\right\}\right) & =m_{i}^{\Theta_{q}}\left(\left\{\omega_{q}\right\}\right) \prod_{p \neq q}^{c} m_{i}^{\Theta_{p}}(\Omega), \forall q \in\{1, \ldots, c\} \\ M_{i}(\Omega) & =\prod_{q=1}^{c} m_{i}^{\Theta_{q}}(\Omega) \\ M_{i}(\emptyset) & =1-\sum_{q=1}^{c} M_{i}\left(\left\{\omega_{q}\right\}\right)-M_{i}(\Omega)\end{cases}
$$

and change the first term of 10$\}$ as $\frac{1}{N} \sum_{i=1}^{N} \sum_{q=1}^{c}\left\{M_{i}\left(\left\{\omega_{q}\right\}\right)-t_{i, q}\right\}^{2}$.

\section{Conclusion}

A new framework for PET imaging based cancer treatment outcome prediction has been proposed in this paper. Features have been extracted from multi-sources of information, which include PET images acquired before and during the treatment, clinical characteristics, and gene expression files. Based on our previous work (Lian et al., 2015a), an improved EFS with prior knowledge and data balancing has been proposed to robustly determine the most informa- 
feature selection, the EK-NN classifier has been trained to predict the outcome. The new prediction system has been evaluated by three clinical studies, showing promising performance with respect to feature selection and classification.

In the future, to further improve the reliability of our prediction system, we plan to include more radiomic features extracted from other image modalities, such as CT, MRI and multi-tracer PETs. In addition, to tackle the imbalanced learning problems, other data balancing or cost-sensitive learning methods should be studied and compared with the method that has been used in this paper.

\section{Appendix A. Radiomic Features Extracted from PET Imaging}

TAble A.7: Definition of SUV-based features. Variable $X$ represents SUVs in the ROI. Function $T[\cdot]$ is a binary indicator. It equals to 1 iff the argument is true. Function $f$ maps $X$ to $L=\{$ tumor,non-tumor $\}$ according to the threshold $40 \% \mathrm{SUV}_{\max }$. Operation $|\cdot|$ calculates the number of voxels within a region.

\begin{tabular}{|c|c|c|}
\hline Feature & Calculation & Description \\
\hline $\mathrm{SUV}_{\max }$ & $\alpha=\max (X)$ & Maximum uptake in the ROI \\
\hline $\mathrm{SUV}_{\text {mean }}$ & $\mu=\operatorname{mean}(X)$ & Average uptake in the ROI \\
\hline $\mathrm{SUV}_{\text {peak }}$ & $\mu_{\alpha}=\frac{1}{\left|N_{\alpha}\right|} \sum_{x \in N_{\alpha}} x$ & $\begin{array}{l}\text { Average uptake in the neighborhood } \\
(3 \times 3 \times 3) \text { of the } \mathrm{SUV}_{\max }\end{array}$ \\
\hline MTV & $\tau=\operatorname{sum}(T[f(X)])$ & Metabolic tumor volume \\
\hline TLG & $\nu=\mu \times \tau$ & Total lesion glycolysis \\
\hline
\end{tabular}


TABle A.8: Definition of GLSZM-based features (Thibault et al. 2014). Let $P$ be the matrix with size $M \times N$. Scalar $R=\sum_{i=1}^{M} \sum_{j=1}^{N} P(i, j)$. Each element $p(i, j)=P(i, j) / R$.

\begin{tabular}{|c|c|c|}
\hline Feature & Calculation & Description \\
\hline Small Zone Emphasis & $\sum_{i}^{M} \sum_{j}^{N} \frac{p(i, j)}{j^{2}}$ & Distribution of small zones. \\
\hline Large Zone Emphasis & $\sum_{i}^{M} \sum_{j}^{N} j^{2} p(i, j)$ & Distribution of large zones. \\
\hline $\begin{array}{l}\text { Low Gray Level Zone } \\
\text { Emphasis }\end{array}$ & $\sum_{i}^{M} \sum_{j}^{N} \frac{p(i, j)}{i^{2}}$ & $\begin{array}{l}\text { Distribution of low gray } \\
\text { level values. }\end{array}$ \\
\hline $\begin{array}{l}\text { High Gray Level Zone } \\
\text { Emphasis }\end{array}$ & $\sum_{i}^{M} \sum_{j}^{N} i^{2} p(i, j)$ & $\begin{array}{l}\text { Distribution of high gray } \\
\text { level values. }\end{array}$ \\
\hline $\begin{array}{l}\text { Small Zone Low Gray } \\
\text { Level Emphasis }\end{array}$ & $\sum_{i}^{M} \sum_{j}^{N} \frac{p(i, j)}{i^{2} j^{2}}$ & $\begin{array}{l}\text { Joint distribution of small } \\
\text { zones and low gray } \\
\text { level values. }\end{array}$ \\
\hline $\begin{array}{l}\text { Small Zone High Gray } \\
\text { Level Emphasis }\end{array}$ & $\sum_{i}^{M} \sum_{j}^{N} \frac{i^{2} p(i, j)}{j^{2}}$ & $\begin{array}{l}\text { Joint distribution of small } \\
\text { zones and high gray } \\
\text { level values. }\end{array}$ \\
\hline $\begin{array}{l}\text { Large Zone High Gray } \\
\text { Level Emphasis }\end{array}$ & $\sum_{i}^{M} \sum_{j}^{N} \frac{j^{2} p(i, j)}{i^{2}}$ & $\begin{array}{l}\text { Joint distribution of large } \\
\text { zones and high gray } \\
\text { level values. }\end{array}$ \\
\hline $\begin{array}{l}\text { Large Zone Low Gray } \\
\text { Level Emphasis }\end{array}$ & $\sum_{i}^{M} \sum_{j}^{N} i^{2} j^{2} p(i, j)$ & $\begin{array}{l}\text { Joint distribution of large } \\
\text { zones and low gray } \\
\text { level values. }\end{array}$ \\
\hline Gray Level Non-Uniformity & $\sum_{i}^{M}\left(\sum_{j}^{N} p(i, j)\right)^{2}$ & $\begin{array}{l}\text { Similarity of gray level } \\
\text { values inside the ROI. }\end{array}$ \\
\hline Zone Size Non-Uniformity & $\sum_{j}^{N}\left(\sum_{i}^{M} p(i, j)\right)^{2}$ & $\begin{array}{l}\text { Similarity of the size of } \\
\text { zones insied the ROI. }\end{array}$ \\
\hline Zone Percentage & $R /(j p(i, j))$ & $\begin{array}{l}\text { homogeneity and distribution } \\
\text { of zones inside the ROI. }\end{array}$ \\
\hline
\end{tabular}

\section{References}

Aerts, H.J., Velazquez, E.R., Leijenaar, R.T., Parmar, C., Grossmann, P., Cavalho, S., Bussink, J., Monshouwer, R., Haibe-Kains, B., Rietveld, D., et al., 2014. Decoding tumour phenotype by noninvasive imaging using a quantitative radiomics approach. Nature Communications 5. 
Barwick, T.D., Taylor, A., Rockall, A., 2013. Functional imaging to predict tumor response in locally advanced cervical cancer. Current Oncology Reports $15,549-558$.

Bloch, I., 1996. Some aspects of Dempster-Shafer evidence theory for classification of multi-modality medical images taking partial volume effect into account. Pattern Recognition Letters 17, 905-919.

Brooks, F.J., Grigsby, P.W., 2014. The effect of small tumor volumes on studies of intratumoral heterogeneity of tracer uptake. Journal of Nuclear Medicine $55,37-42$.

Calais, J., Thureau, S., Dubray, B., Modzelewski, R., Thiberville, L., Gardin, I., Vera, P., 2015. Areas of high 18F-FDG uptake on preradiotherapy PET/CT identify preferential sites of local relapse after chemoradiotherapy for nonsmall cell lung cancer. Journal of Nuclear Medicine 56, 196-203.

Chawla, N.V., Bowyer, K.W., Hall, L.O., Kegelmeyer, W.P., 2002. SMOTE : synthetic minority over-sampling technique. Journal of Artificial Intelligence Research , 321-357.

Chen, X., Wasikowski, M., 2008. Fast : a ROC-based feature selection metric for small samples and imbalanced data classification problems, in : Proceedings of the 14th ACM SIGKDD International Conference on Knowledge Discovery and Data Mining, ACM. pp. 124-132.

Cook, G.J., Siddique, M., Taylor, B.P., Yip, C., Chicklore, S., Goh, V., 2014. Radiomics in PET : principles and applications. Clinical and Translational Imaging 2, 269-276.

Deep, K., Singh, K.P., Kansal, M., Mohan, C., 2009. A real coded genetic algorithm for solving integer and mixed integer optimization problems. Applied Mathematics and Computation 212, 505-518. 
Denœux, T., 1995. A k-nearest neighbor classification rule based on DempsterShafer theory. IEEE Transactions on Systems, Man and Cybernetics 25, 804-813.

Denœux, T., Smets, P., 2006. Classification using belief functions : relationship between case-based and model-based approaches. IEEE Transactions on Systems, Man, and Cybernetics, Part B : Cybernetics 36, 1395-1406.

Efron, B., Tibshirani, R., 1997. Improvements on cross-validation : the $632+$ bootstrap method. Journal of the American Statistical Association 92, 548560 .

El Naqa, I., Grigsby, P., Apte, A., Kidd, E., Donnelly, E., Khullar, D., Chaudhari, S., Yang, D., Schmitt, M., Laforest, R., et al., 2009. Exploring featurebased approaches in PET images for predicting cancer treatment outcomes. Pattern Recognition 42, 1162-1171.

${ }_{640}$ Guyon, I., Elisseeff, A., 2003. An introduction to variable and feature selection. The Journal of Machine Learning Research 3, 1157-1182.

Guyon, I., Weston, J., Barnhill, S., Vapnik, V., 2002. Gene selection for cancer classification using support vector machines. Machine learning 46, 389-422.

He, H., Garcia, E., et al., 2009. Learning from imbalanced data. IEEE Transactions on Knowledge and Data Engineering 21, 1263-1284.

Kira, K., Rendell, L.A., 1992. The feature selection problem : Traditional methods and a new algorithm, in : Proceedings of the tenth National Conference on Artificial Intelligence (AAAI-92), pp. 129-134.

Lanic, H., Mareschal, S., Mechken, F., Picquenot, J.M., Cornic, M., Maingonnat, 650 C., Bertrand, P., Clatot, F., Bohers, E., Stamatoullas, A., et al., 2012. Interim positron emission tomography scan associated with international prognostic index and germinal center B cell-like signature as prognostic index in diffuse large B-cell lymphoma. Leukemia \& Lymphoma 53, 34-42. 
Lelandais, B., Ruan, S., Denœux, T., Vera, P., Gardin, I., 2014. Fusion of multitracer PET images for dose painting. Medical Image Analysis 18, 1247-1259.

Lemarignier, C., Di Fiore, F., Marre, C., Hapdey, S., Modzelewski, R., Gouel, P., Michel, P., Dubray, B., Vera, P., 2014. Pretreatment metabolic tumour volume is predictive of disease-free survival and overall survival in patients with oesophageal squamous cell carcinoma. European Journal of Nuclear Medicine and Molecular Imaging 41, 2008-2016.

Lian, C., Ruan, S., Denœux, T., 2015a. An evidential classifier based on feature selection and two-step classification strategy. Pattern Recognition 48, 23182327 .

Lian, C., Ruan, S., Denoux, T., Vera, P., 2015b. Outcome prediction in tumour therapy based on Dempster-Shafer theory, in : Biomedical Imaging (ISBI), 2015 IEEE 12th International Symposium on, IEEE. pp. 63-66.

Liu, Z.G., Pan, Q., Dezert, J., Mercier, G., 2015. Credal c-means clustering method based on belief functions. Knowledge-Based Systems 74, 119-132.

Makni, N., Betrouni, N., Colot, O., 2014. Introducing spatial neighbourhood in evidential c-means for segmentation of multi-source images : application to prostate multi-parametric MRI. Information Fusion 19, 61-72.

Masson, M.H., Denœux, T., 2008. ECM : An evidential version of the fuzzy c-means algorithm. Pattern Recognition 41, 1384-1397.

Mi, H., Petitjean, C., Dubray, B., Vera, P., Ruan, S., 2015. Robust feature selection to predict tumor treatment outcome. Artificial Intelligence in Medicine $64,195-204$.

Murphy, K., van Ginneken, B., Schilham, A.M., De Hoop, B., Gietema, H., Prokop, M., 2009. A large-scale evaluation of automatic pulmonary nodule detection in chest $\mathrm{CT}$ using local image features and k-nearest-neighbour classification. Medical Image Analysis 13, 757-770. 
Ou, Y., Shen, D., Zeng, J., Sun, L., Moul, J., Davatzikos, C., 2009. Sampling the spatial patterns of cancer : Optimized biopsy procedures for estimating prostate cancer volume and gleason score. Medical Image Analysis 13, 609620 .

Ou, Y., Sotiras, A., Paragios, N., Davatzikos, C., 2011. DRAMMS : Deformable registration via attribute matching and mutual-saliency weighting. Medical Image Analysis 15, 622-639.

Prastawa, M., Bullitt, E., Ho, S., Gerig, G., 2004. A brain tumor segmentation framework based on outlier detection. Medical Image Analysis 8, 275-283.

Pudil, P., Novovičová, J., Kittler, J., 1994. Floating search methods in feature selection. Pattern Recognition Letters 15, 1119-1125.

Shafer, G., 1976. A mathematical theory of evidence. Princeton University Press.

Smets, P., Kennes, R., 1994. The transferable belief model. Artificial Intelligence $66,191-234$.

Somol, P., Novovicova, J., 2010. Evaluating stability and comparing output of feature selectors that optimize feature subset cardinality. IEEE Transactions on Pattern Analysis and Machine Intelligence 32, 1921-1939.

Tan, S., Kligerman, S., Chen, W., Lu, M., Kim, G., Feigenberg, S., D’Souza, W.D., Suntharalingam, M., Lu, W., 2013. Spatial-temporal [18 F]FDG-PET features for predicting pathologic response of esophageal cancer to neoadjuvant chemoradiation therapy. International Journal of Radiation Oncology* Biology* Physics 85, 1375-1382.

Thibault, G., Angulo, J., Meyer, F., 2014. Advanced statistical matrices for texture characterization : application to cell classification. IEEE Transactions on Biomedical Engineering 61, 630-637. 
Tixier, F., Le Rest, C.C., Hatt, M., Albarghach, N., Pradier, O., Metges, J.P., Corcos, L., Visvikis, D., 2011. Intratumor heterogeneity characterized by textural features on baseline 18F-FDG PET images predicts response to concomitant radiochemotherapy in esophageal cancer. Journal of Nuclear Medicine $52,369-378$.

Wang, F., Miron, A., Ainouz, S., Bensrhair, A., 2014. Post-aggregation stereo matching method using Dempster-Shafer theory, in : 2014 IEEE International Conference on Image Processing (ICIP 2014), IEEE. pp. 3783-3787.

Wang, G., Zhang, S., Xie, H., Metaxas, D.N., Gu, L., 2015. A homotopybased sparse representation for fast and accurate shape prior modeling in liver surgical planning. Medical Image Analysis 19, 176-186.

Wang, L., 2008. Feature selection with kernel class separability. IEEE Transactions on Pattern Analysis and Machine Intelligence 30, 1534-1546.

${ }_{720}$ Xu, P., Davoine, F., Bordes, J.B., Zhao, H., Denœux, T., 2014. Multimodal information fusion for urban scene understanding. Machine Vision and Applications , 1-19.

Yager, R.R., 1987. On the Dempster-Shafer framework and new combination rules. Information Sciences 41, 93-137.

Zhang, N., Ruan, S., Lebonvallet, S., Liao, Q., Zhu, Y., 2011. Kernel feature selection to fuse multi-spectral MRI images for brain tumor segmentation. Computer Vision and Image Understanding 115, 256-269.

Zhu, H., Basir, O., 2005. An adaptive fuzzy evidential nearest neighbor formulation for classifying remote sensing images. IEEE Transactions on Geoscience and Remote Sensing 43, 1874-1889.

Zouhal, L.M., Denœux, T., 1998. An evidence-theoretic k-NN rule with parameter optimization. IEEE Transactions on Systems, Man, and Cybernetics, Part C : Applications and Reviews 28, 263-271. 